\title{
Thumb and vallecula signs in acute infectious epiglottitis
}

\author{
Hiroki Matsuura MD, Takashi Fukumura MD
}

— Cite as: CMAJ 2017 October 16;189:E1289. doi: 10.1503/cmaj.170194

A 66-year-old woman presented to the emergency department with a one-day history of acute-onset severe throat pain, hoarseness, and dyspnea. She did not have any previous respiratory problems or history of allergy.

On examination the patient had stridor and a muffled voice, along with drooling. Radiography of the lateral soft tissue of the neck showed an enlarged and swollen epiglottis, and obliteration of the vallecula. These findings were consistent with the "thumb sign" and "vallecula sign," respectively, suggestive of an acute infectious epiglottitis or supraglottitis (Figure 1A). Flexible fibreoptic nasopharyngolaryngoscopy showed substantial pooling of secretions, a diffusely edematous epiglottis, and aryepiglottic folds that obstructed the airway (Figure 1B). We performed an urgent tracheostomy under local anesthesia for airway management. The patient was treated with ceftriaxone, clindamycin and betamethasone.

Five days after intubation, laryngoscopy showed resolution of the swelling in the patient's epiglottis, and she was successfully extubated. Results from blood culture tests were negative after several days. The patient made a full recovery and was discharged from the hospital, with antibiotics (orally administered) and outpatient follow-up.

Epiglottitis or supraglottitis is an infection of the supraglottic region of the oropharynx, including epiglottis, vallecula and aryepiglottic folds. In general, symptoms progress rapidly and lead to dysphagia, drooling, trismus, stridor and dyspnea. Although infectious epiglottitis was historically most often caused by Haemophilus influenzae serotype $b$ (Hib), the success of widespread Hib vaccination has reduced the incidence of this disease among children. ${ }^{1}$ Infectious epiglottitis caused by Hib is becoming more common in adults than in children. However, the most common bacteria causing infectious epiglottitis in adults currently are Streptococcus pyogenes, Streptococcus pneumoniae and Staphylococcus aureus. ${ }^{2}$ Therefore, clinicians should select antibiotics to which these bacteria are susceptible.

Adult infectious epiglottitis may be unpredictable and difficult to diagnose definitively. If epiglottitis is suspected on the basis of clinical features, and the airway is judged to be at risk, timely airway intervention should not be delayed by attempts to obtain radiographs or perform culture tests. ${ }^{3}$ Fibre-optic examination may precipitate acute dyspnea and should only be undertaken where immediate rescue facilities are available. ${ }^{4}$

\section{References}

1. Briere EC, Rubin L, Moro PL, et al.; Division of Bacterial Diseases, National Center for Immunization and Respiratory Diseases, CDC. Prevention and control of Haemophilus influenzae type b disease: recommendations of the Advisory Committee on Immunization Practices (ACIP). MMWR Recomm Rep 2014;63:1-14.

2. Sivakumar S, Latifi SQ. Varicella with stridor: think group A streptococcal epiglottitis. J Paediatr Child Health 2008;44:149-51.

3. Ames WA, Ward VM, Tranter RM, et al. Adult epiglottitis: an under-recognized, life-threatening condition. Br J Anaesth 2000;85:795-7.

4. Glynn F, Fenton JE. Diagnosis and management of supraglottitis (epiglottitis). Curr Infect Dis Rep 2008;10:200-4.

\section{Competing interests: None declared.}

This article has been peer reviewed.

The authors have obtained patient consent.

Affiliation: Mitoyo General Hospital, Himehama, Toyohama-cho, Kanonji-city, Kagawa, Japan

Correspondence to: Hiroki Matsuura, superonewex0506@yahoo.co.jp 